\title{
Poincaré's Recurrence Theorem and the Unitarity of the $S$-Matrix
}

\author{
A. M. Ozorio de Almeida and R. O. Vallejos \\ Centro Brasileiro de Pesquisas Fúsicas \\ Rua Xavier Sigaud 150, 22290-180 Rio de Janeiro, Brazil
}

(July 22, 2018)

\begin{abstract}
A scattering process can be described by suitably closing the system and considering the first return map from the entrance onto itself. This scattering map may be singular and discontinuous, but it will be measure preserving as a consequence of the recurrence theorem applied to any region of a simpler map. In the case of a billiard this is the Birkhoff map. The semiclassical quantization of the Birkhoff map can be subdivided into an entrance and a repeller. The construction of a scattering operator then follows in exact analogy to the classical process. Generically, the approximate unitarity of the semiclassical Birkhoff map is inherited by the $S$-matrix, even for highly resonant scattering where direct quantization of the scattering map breaks down.
\end{abstract}

PACS numbers: 05.45.+b, 03.65.Sq, 03.80.+r

For a classical conservative system, whether discrete or continuous in time, Poincaré's celebrated theorem can be reduced to the general statement that the probability for an orbit to return to any given region is unity if the motion is bounded. There is no restriction on the time this recurrence will take, which may vary widely among orbits starting in different subregions. In any case, by waiting a sufficiently long time, the first return of each trajectory defines a measure preserving map of the region onto itself.

The essential boundedness of the system in no way hinders its application to classical scattering problems, because we can always choose the appointed region to coincide with the opening of the scattering system to the outside world. Since we are only interested in the first return of the orbits to the chosen region, it makes no difference that the system is not really closed. In other words, we can still apply the theorem if the union of the scatterer and the opening combine to form a bounded measure preserving map.

As a first example consider the simple scattering system composed of a circular billiard opening onto a straight tube [1], as shown in Fig. 1. In this case, the closure of the dynamical system can be reduced to the Birkhoff map (or bounce map) for specular collisions of the straight trajectories with the circular boundary. The phase space is defined by the boundary coordinate $s$ (or the angle $\theta$, in the case of unit radius) and $p_{s}$, the tangential momentum (proportional to $\cos \alpha$, where $\alpha$ is the angle of incidence).

The closed dynamics is very simple in this case: $p_{s}$ is constant (integrable motion) and $\Delta \theta=2 \alpha$. Nonetheless, the scattering map of the orbits returning to the opening is discontinuous. Indeed it is composed of an infinite sequence of diminishing subregions, of which the first few are shown in Fig. 2. Therefore a maximally simple closed dynamics induces a relatively complex (resonant) scattering map. It is only in the (non resonant) limit where the size of the opening approaches the diameter of the circle that the scattering map is also simple.

Consider now the less obvious example of the specular scattering from three disks, that has become the paradigm of chaotic classical scattering [2]. It may appear that our choice of closing surface in Fig. 3 amounts to an overkill, since we are not interested in orbits such as a in Fig. 3. However this integrable motion described in the previous example does not mix with the scattering orbits such as $\mathbf{b}$, so we can substract it from the phase space of our system. This is then composed of the Birkhoff coordinates of the external circuit, restricted to small $p_{s}$ added to the full Birkhoff coordinates of the three scattering circles, as shown in Fig. 4. Evidently, we obtain the useful asymptotic scattering picture by making the radius of the outer circle arbitrarily large, so that we can identify the exit direction of an orbit with the point where it collides with the outer circle. Even so, the useful area for the outer circle in the phase space of Fig. 4 will be smaller than that of the three disks combined.

The dynamics for the first return of the closed map is indicated by the different regions in Fig. 4. This is less trivial than in the previous example, but it is nowhere singular. The first return map between the four circles is hyperbolic and discontinuous similarly to the baker's transformation [3], but the full complexity of the motion only arises through the multiple iterations needed to compose the scattering map of first returns to the outer circle. This map exhibits a fractal structure of singularities generated by motion that nearly enters on the stable manifold of periodic orbits within the scatterer 任.

In both our simple examples it may still be necessary to relate the map restricted to the opening to a map purely determined by the mesurement to be performed. In the case of the circle that opens onto the tube, one is finally concerned with the orbits entering and leaving the other end of the tube, whereas in the three disc case we should connect the enclosing circle to an asymptotically large circle. The resulting map is known as the Poincaré scattering map [5]. Our immediate concern will be the quantization of the (first return) internal map rather than the details of its outer connections. 
An evident conceptual advantage is achieved by understanding the structure of scattering maps on the basis of multiple iterations of their closure, even though it may be necessary to reverse this procedure in experimental situations. Our purpose is now to show that we can transfer to semiclassical scattering a construction of open and closed systems corresponding to the one which we have been employing in the classical theory.

The starting point is to note that we can always define a finite Hilbert space that will correspond semiclassically to a finite phase space [6]. Indeed, the dimension of the Hilbert space corresponding to a two dimensional classical phase space of area $A$ will be $N=A / 2 \pi \hbar$, where $\hbar$ is Planck's constant. The prescription given by Miller [7] for the approximately unitary quantum map $U$, is given in the coordinate representation as

$$
U\left(q, q^{\prime}\right) \simeq \frac{1}{\sqrt{2 \pi \hbar}} \sum_{j}\left|\frac{\partial^{2} \sigma_{j}}{\partial q \partial q^{\prime}}\right|^{1 / 2} e^{i \sigma\left(q, q^{\prime}\right) / \hbar+i \mu_{j}},
$$

where $\mu_{j}$ is the Maslov phase and $\sigma_{j}\left(q, q^{\prime}\right)$ stands for the generating function of the classical map, given implicitly by

$$
p^{\prime}=\frac{\partial \sigma_{j}}{\partial q^{\prime}}, \quad p=-\frac{\partial \sigma_{j}}{\partial q},
$$

the index $j$ indicating that there may be more than one orbit for a given pair of points $\left(q^{\prime}, q\right)$. Of course, we should worry about the discretization of coordinates, due to the finite dimension $(N)$ of the Hilbert space, but the approximations will hold when $N$ is large. Furthermore, the semiclassical approximation (11) will leave out evanescent modes [8] and diffraction effects (e.g. in the three disks problem), but again these will give relatively small contributions in the large $N$ limit.

We can check the approximate unitarity of (11) by noting that, for each continuous region of area $A_{j}$

$$
N_{j}=\operatorname{Tr} U_{j} U_{j}^{\dagger} \simeq \int \frac{d q d q^{\prime}}{2 \pi \hbar}\left|\frac{\partial^{2} \sigma_{j}}{\partial q \partial q^{\prime}}\right|=\frac{A_{j}}{2 \pi \hbar} .
$$

Thus, we are quantizing separately each subregion in a way that increases border effects for discontinuous maps with many subdivisions. We have shown that this is typical of scattering maps. If they are sufficiently resonant as in our examples, we obtain $N_{j} \lesssim 1$ for many subregions, which are hence beyond the range of validity of the Miller prescription. The quantum signature of this resonance problem is the need to account for a large number of evanescent modes that cannot be related to real classical orbits.

It is important to note that a given scattering experiment may involve the amplitude of a single element of the $S$-matrix in the appropriate representation. We thus require detailed local knowledge of this operator, rather than merely its traces or other coarse grained information. Experience with the quantized baker's map and other discontinuous maps analogous to the present scattering situation, shows that an iteration of the map may have a reasonable semiclassical approximation for its trace beyond the time when the semiclassical approximation for the full map has broken down [9]. Moreover, we are not concerned with the small departure from unitarity of the Miller construction for a continuous classical map. Instead we seek to remedy its complete breakdown for highly resonant scattering maps.

The way out is to rely on the construction of the scattering map from the multiple iterations of the simpler closed map. We thus need the following result, which may be considered as the quantization of the recurrence thorem:

Given a finite Hilbert space $H_{N}$ subdivided into two orthogonal subspaces $H_{N_{0}}=P_{0} H_{N}$ and $H_{N_{1}}=P_{1} H_{N}$ (such that the projection operators $P_{0}+P_{1}=1_{N}$ ) and given an unitary operator $U_{N}$ defined on $H_{N}$, then the operator

$$
S_{N_{0}}=P_{0} U_{N}\left[1-P_{1} U_{N}\right]^{-1} P_{0}=P_{0} U_{N} \sum_{m=0}^{\infty}\left(P_{1} U_{N}\right)^{m} P_{0}
$$

is unitary on $H_{N_{0}}$. It should be noted that this is a much stronger property than the obvious "weak unitarity" statement

$$
\forall \psi_{0} \in H_{N_{0}}:\left|\psi_{0}\right|^{2}=\sum_{m=0}^{\infty}\left|P_{0} U_{N}\left(P_{1} U_{N}\right)^{m} \psi_{0}\right|^{2},
$$

which corresponds to the overall conservation of classical probability. To outline the proof of the theorem, we define the rectangular blocks of the operator $U_{N}$, namely $U_{i j}=P_{i} U P_{j}$, and their hermitian conjugates $U_{i j}^{\dagger}=P_{j} U^{\dagger} P_{i}$. The unitarity of $U_{N}$ implies

$$
\begin{aligned}
& U_{00} U_{00}^{\dagger}+U_{01} U_{10}^{\dagger}=1_{N_{0}} \\
& U_{00} U_{01}^{\dagger}+U_{01} U_{11}^{\dagger}=0 \\
& U_{10} U_{01}^{\dagger}+U_{11} U_{11}^{\dagger}=1_{N_{1}},
\end{aligned}
$$

where $1_{N_{j}}$ coincides with the nonsingular block of $P_{j}$. In this notation Eq. (4) reads $S_{N_{0}}=U_{00}+U_{01}\left[1_{N_{1}}-\right.$ $\left.U_{11}\right]^{-1} U_{10}$. Then it is straightforward, though a little lengthy, to verify that $S_{N_{0}} S_{N_{0}}^{\dagger}=S_{N_{0}}^{\dagger} S_{N_{0}}=1_{N_{0}}$ is a consequence of Eqs. (6-8).

Consider now a basis for $H_{N_{1}}$ in which $P_{1} U$ is diagonal, with states $\psi_{1}^{j}$, and the corresponding eigenvalues $\lambda_{1}^{j}$. If the elements of $U$ that couple such a state to the subspace $H_{N_{0}}$ are labeled $U_{10}^{j k}$, then

$$
\left|\lambda_{1}^{j}\right|^{2}=1-\sum_{k}\left|U_{10}^{j k}\right|^{2} .
$$


It follows that each eigenvalue of $P_{1} U$ lies inside the unit circle, unless the corresponding eigenstate is completely uncoupled to $H_{N_{0}}$ (in which case this eigenstate should be substracted from $H_{N_{1}}$ ). The quantum map $P_{1} U$ is therefore strictly dissipative, corresponding to the classical scatterer that looses orbits at each iteration 10]. This fact is essential for the convergence of the sum in (位) of the expansion for $S_{N_{0}}$.

We can now apply the exact result (4) to scattering by identifying $U_{N}$ with the approximate semiclassical map (11) for the closure of the scattering system. The resulting scattering matrix given by (11) determines the on-shell $S$ matrix for fixed energy 11.

If the semiclassical approximation for $U$ departs from unitarity by order $\epsilon$, the scattering matrix will err by an order $\epsilon\left[1-U_{11}(\epsilon)\right]^{-2}$. So there could be a large deviation of the $S$-matrix from unitarity if one of the eigenvalues of $U_{11}$ were sufficiently close to the unit circle. However, $\epsilon$ vanishes as $\hbar \rightarrow 0$, whereas the coupling of the scatterer to the opening is classically strong in our examples, so that (9) guarantees that asymptotically $\left[1-U_{11}(\epsilon)\right]^{-2}$ remains finite. Therefore we can use the quantum recurrence theorem as a basis for the semiclassical approximation of the scattering matrix.

In our examples, the energy dependence of the classical map is trivial and it can be scaled away, but the area of the phase space grows with energy, modifying the dimension of the corresponding Hilbert space. Another way to see this important energy dependence of the quantum mechanics is through the growth of the actions of the orbits, i.e. the generating functions $\sigma_{j}$ in (ID). In the case of smooth potentials, rather than billiards, even the energy dependence is nontrivial, but these scattering systems can also be treated within our conceptual framework by introducing quantum Poincaré sections in the manner of Bogomolny [6] or Rouvinez and Smilansky [12].

Formally, we could reobtain the standard Miller formula for the scattering amplitudes by doing the matrix multiplications in the infinite expansion (画) by the method of stationary phase. If we keep the semiclassical representation (11) for the unitary matrix $U$ and compute $\left[1_{N_{1}}-U_{11}\right]^{-1}$ using "Fredholm Theory", we rederive precisely the semiclassical scattering theory of Georgeot and Prange 13]. Our approach shows that each of the operators employed in the semiclassical Fredholm theory originates in the quantization of Poincaré's recurrence theorem, as well as elucidates their approximate unitary or dissipative character.

Finally, our results allow for a minimal semiclassical approximation for the scattering matrix. The only approximation is the use of Miller's theory for the global map $U_{N}$ (rather than for the scattering map, as in previous treatments), using the classical variables corresponding to the entrance and exit channels. The problem is then reduced to the numerical inversion of the matrix $\left[1_{N_{1}}-U_{11}\right]$, which has the dimension of the classical phase space of the scatterer divided by Planck's constant.

\section{ACKNOWLEDGMENTS}

We would like to thank C. Anteneodo and R. Markarian for useful suggestions. This work was supported by the Conselho de Desenvolvimento Científico e Tecnológico $(\mathrm{CNPq} /$ Brazil).

[1] R. Blümel, B. Dietz, C. Jung, and U. Smilansky, J. Phys. A 25, 1483 (1992).

[2] B. Eckhardt, G. Russberg, P. Cvitanović, P. E. Rosenqvist, and P. Scherer, in Quantum Chaos-Between Order and Disorder, G. Casati and B. Chirikov, Eds. (Cambridge University Press, Cambridge, 1995).

[3] N. L. Balazs and A. Voros, Ann. Phys. (NY) 190, 1 (1989).

[4] J. H. Jensen, Phys. Rev. Lett. 73, 244 (1994).

[5] C. Jung and T. H. Seligman, Phys. Rep. 285, 77 (1997).

[6] E. Bogomolny, Nonlinearity 5, 805 (1992).

[7] W. H. Miller, Adv. Chem. Phys. 25, 69 (1974).

[8] E. Doron and U. Smilansky, Nonlinearity, 5, 1055 (1992); J. E. Ortiz and A. M. Ozorio de Almeida, J. Phys. A (to appear).

[9] M. G. E. da Luz and A. M. Ozorio de Almeida, Nonlinearity 8, 43 (1995), and Physica D 94, 1 (1996).

[10] Maps of this kind have been quantized in M. Saraceno and R. O. Vallejos, CHAOS 6, 193 (1996).

[11] Even though our formula for $S_{N_{0}}$ strongly resembles some standard constructions of scattering theory (e.g. Feshbach's projector formalism [H. Feshbach, Ann. Phys. 19, 287 (1962); C. H. Lewenkopf and H. A. Weidenmüller, Ann. Phys. 212, 53 (1991)]), at this stage we have only a prescription for generating an unitary operator.

[12] C. Rouvinez and U. Smilansky, J. Phys. A 28, 77 (1995).

[13] B. Georgeot and R. E. Prange, Phys. Rev. Lett. 74, 4110 (1995). 


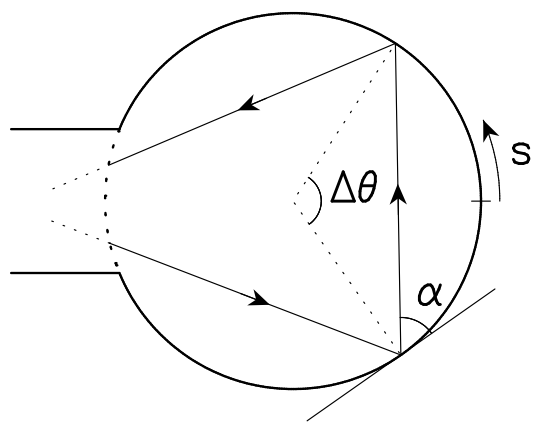

Fig. 1 * Ozorio de Almeida \& Vallejos

FIG. 1. Scattering by a circular cavity. A particle propagates from the asymptotic region (straight tube) up to the entrance of the interaction region (dotted arc, approximating a straight segment). This defines an initial condition for the internal map of specular reflections against the circular boundary. Eventually, the particle returns to the opening, entering the tube instead of reflecting again. The coordinates for the internal bounce map are $s$, the distance along the boundary, and the tangent momentum $p_{s}$, proportional to $\cos \alpha$. The rule for specular reflections is $\Delta \theta=2 \alpha$.

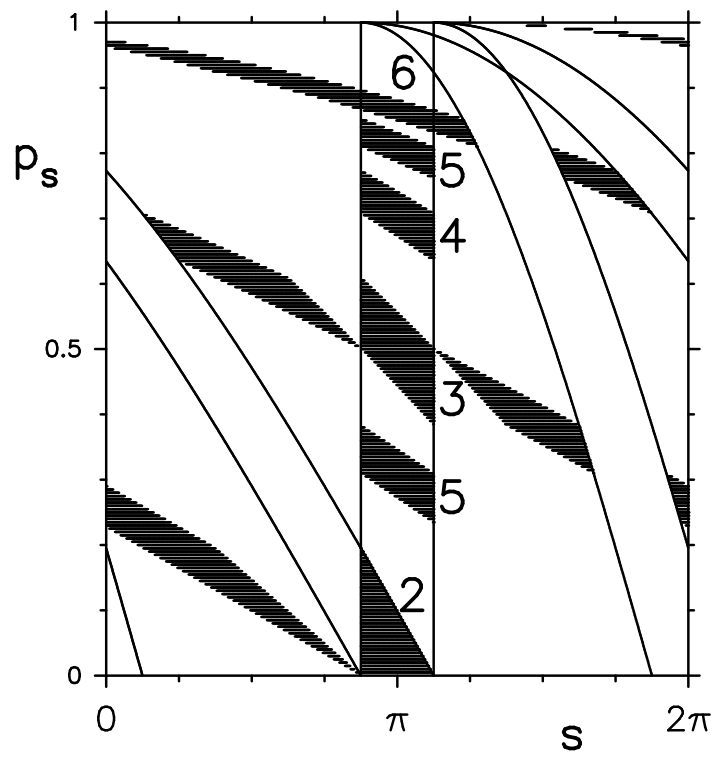

Fig. $2 *$ Ozorio de Almeida \& Vallejos
FIG. 2. The first-return map for the circular system of Fig. 1. Initial conditions having all possible momenta are launched from the entrance of the scattering system. In the Birkhoff plane $s-p_{s}$, this corresponds to the narrow rectangular region centered at $s=\pi$. We show the first two images of this region by the full internal map (strips bounded by the cosine curves). Also shown are the first returns after $2,3, \ldots, 6$ bounces with the circular boundary, and the phase points that still stay inside the cavity after 6 bounces (dark strips ouside the initial rectangle). We have not plotted those points that return after one iteration as they do not correspond to the scattering process (for the system closed by a straight segment there would be no orbits leaving at the first return). The picture for $p_{s}<0$ is obtained by reflection with respect to the point $\left(s=\pi, p_{s}=0\right)$.

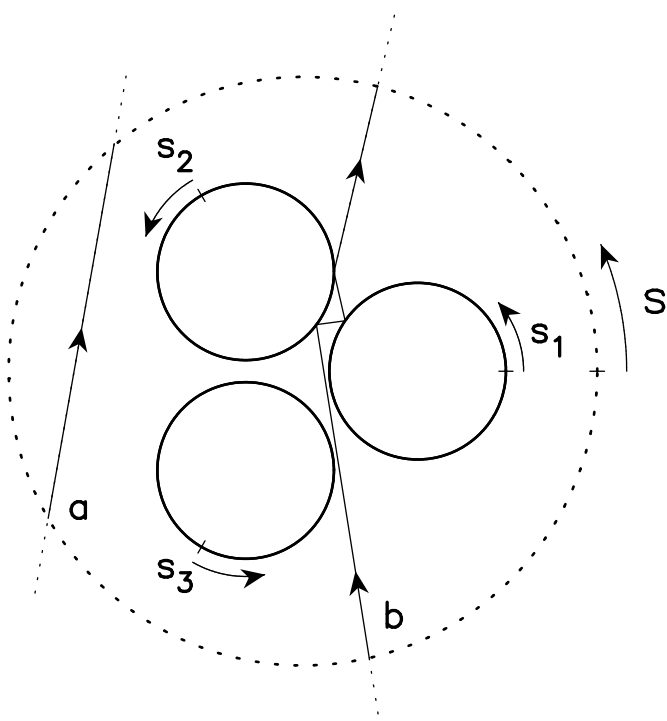

Fig. $3 *$ Ozorio de Almeida \& Vallejos

FIG. 3. Scattering by three discs. An incident particle propagates trivially to the outer circle that closes the system. This defines an initial condition for the Birkhoff map of the closed problem, described by the full set of position coordinates $\left\{s, s_{1}, s_{2}, s_{3}\right\}$ together with the tangent momenta. After colliding with the discs a certain number of times, the particle returns to the outer circle, i.e. it escapes. 
(a)
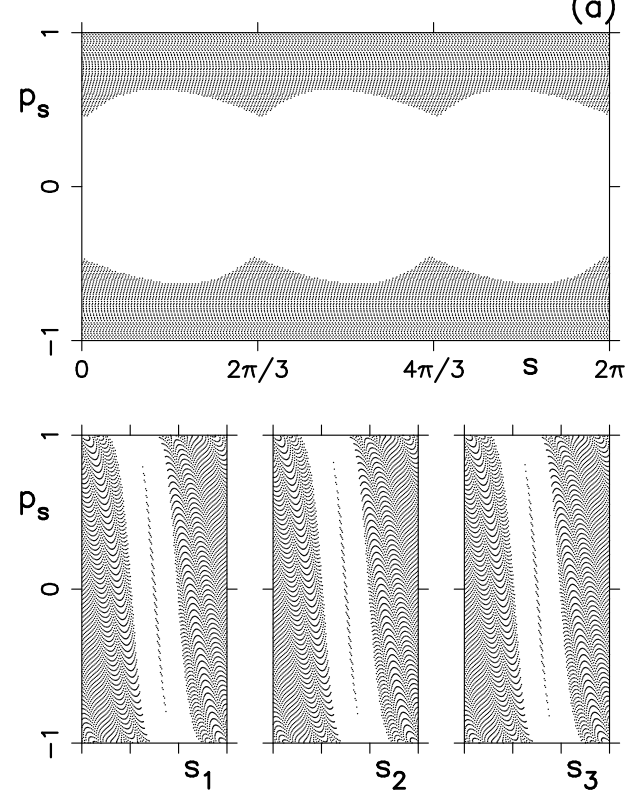

Fig. 4a* Ozorio de Almeida \& Vallejos

(b)
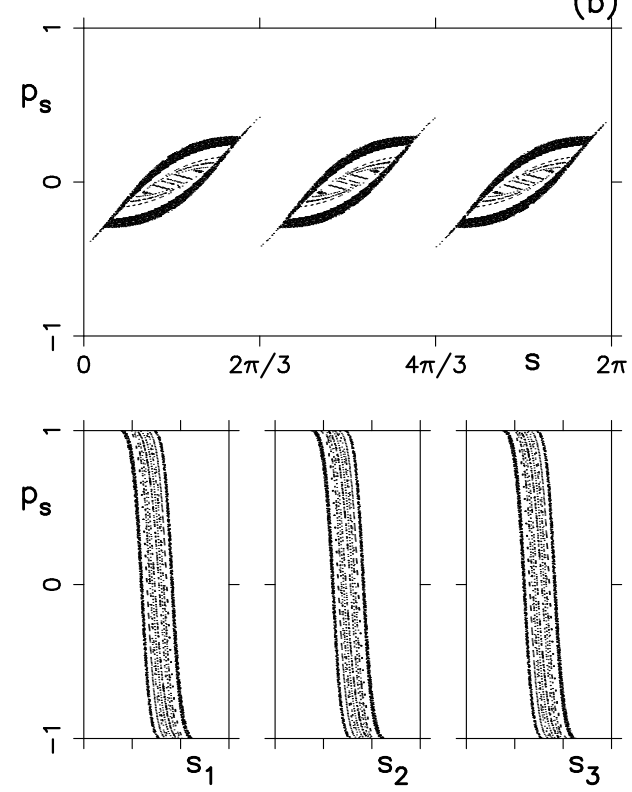

Fig. 4b * Ozorio de Almeida \& Vallejos
FIG. 4. The return map for the three-discs system. Initial conditions are started at the outer circle of Fig. 3, with momenta pointing inwards (upper rectangles). (a) The first iterate of the initial rectangle is shown in dark. Accordingly, white regions correspond to the first iterate of the phase space of the three discs. The dark region in the phase space of the outer circle is associated to scattering trajectories that do not hit the inner discs (indicated as a in Fig. 3). (b) After three iterations fractal structures begin to develop. Shown are those trajectories-stuck to the discs-that have not escaped after three bounces (like b in Fig. 3), and those which are escaping after two bounces (upper rectangle). 